\title{
A Multicriteria Framework for Cloud Service Providers Selection Based on the Matter Element Extension Method
}

\author{
Constanţa Zoie RADULESCU ${ }^{1 *}$, Marius RADULESCU ${ }^{2}, \operatorname{Radu}_{\text {BONCEA }}{ }^{1,3}$, \\ Ionut PETRE ${ }^{1,4}$, Ionut-Eugen SANDU ${ }^{1,4}$, Mihail DUMITRACHE ${ }^{1,5}$ \\ ${ }^{1}$ National Institute for Research and Development in Informatics - ICI Bucharest, \\ 8-10 Maresal Averescu Avenue, Bucharest, 01145, Romania \\ zoie.radulescu@ici.ro (*Corresponding author), radu.boncea@ici.ro, ionut.petre@ici.ro, \\ ionut.sandu@ici.ro, mihail.dumitrache@ici.ro \\ 2 "Gheorghe Mihoc-Caius Iacob" Institute of Mathematical Statistics and Applied Mathematics of the \\ Romanian Academy, Calea 13 Septembrie, No.13, Bucharest, 050711, Romania \\ mradulescu.csmro@yahoo.com \\ ${ }^{3}$ Politehnica University of Bucharest, Splaiul Independentei, No. 313, Bucharest, 60042, Romania \\ 4 "Lucian Blaga" University of Sibiu, 10 Victoriei Blvd., Sibiu, 550024 Romania \\ ${ }^{5}$ University of Bucharest - Faculty of Letters, 5-7 Edgar Quinet Street, Bucharest, 010017, Romania
}

\begin{abstract}
Market availability of multiple cloud services with similar characteristics, various pricing structures, and different Quality of Service (QoS) criteria make the selection of a Cloud Service Provider (CSP) a difficult task for decision makers that want to move their business to the cloud. CSP selection is a complex multicriteria problem. Many of the QoS criteria that must be considered in this problem are both quantitative and qualitative criteria. This fact determines the inclusion in the decision process of the uncertainty due to the subjectivism of the qualitative criteria evaluation. The present paper proposes a new multicriteria framework based on the Matter Element Extension (MEE) method to solve the problem of ranking a set of CSPs and the selection of the CSP that best meets the requirements of an organization. MEE method is ideal to solve multicriteria problems by formalizing the decision process. It can be enhanced by combining it with weighting methods. The multicriteria framework based on MEE method is implemented in a case study. A sensitivity analysis is performed by varying the endpoints of the extended intervals for criteria. The solutions and the CSP ranks obtained are analysed.
\end{abstract}

Keywords: Multicriteria framework, Cloud Services Providers, Matter Element Extension method, Ranking, Classic and extended intervals, Sensitivity analysis.

\section{Introduction}

In the last years, there has been a rapid growth of cloud computing technologies that has led to a massive increase in the number of Cloud Service Providers (CSPs) that supply of a wide range of cloud services.

Cloud computing and service providing has been deployed, over the recent years, as a promising enabling technology for many domains such as network control / cloud control systems (see, for example, (Mahmoud \& Hamdan, 2018; Xia, 2015)), decision-making (see, for example, (Martens \& Teuteberg, 2012; Filip et al., 2017)) and so on. In each case, the set of criteria and the associated weights are context-dependent, and, eventually, influence the adoption of the solution offered by a method.

The availability, on the market, of multiple cloud services with similar features, various pricing structures, and different Quality of Service (QoS) regulations make the selection of a CSP a difficult task for decision makers, in organizations, that are looking to move their business to the cloud. In addition, decision-makers must consider multiple quantitative and qualitative criteria that are often conflicting when making a choice. Because a decision maker must consider several QoS criteria when choosing a CSP, from a set of CSPs considered as a set of alternatives, this issue falls within the domain of the Multicriteria Making Decision (MCDM) (Hussain et al., 2020a).

There are numerous researches in the literature related to solving the problem of CSP ranking and selection, through multicriteria decision methods. Some MCDM solutions for solving this problem, proposed in literature, are presented in the following. In (Abdel-Basset et al., 2018) a neutrosophic multi-criteria decision analysis approach is provided for estimating the CSP quality. In the paper (Tiwari \& Kumar, 2020) a new framework is proposed to identify security and privacy requirements and select an appropriate CSP. The paper (Al-Faifi et al., 2019) proposed a method to help the organizations to select the appropriate CSP which can fulfil their requirements. A hybrid multicriteria decision method based on a combination of a k-means algorithm and the DEMATEL-ANP method was 
developed to evaluate and rank a set of CSPs from Smart data. A new selection model, which was deployed on Amazon cloud platform, was presented in (Eisa et al., 2020). The proposed model was implemented as a simulation tool. The paper (Halabi \& Bellaiche, 2018) presents a model that addresses to the dynamic CSPs ranking and selection. The problem is solved using a Linear Programming Model.

In the paper (Alam et al., 2018), is developed an integrated decision model based on the Fuzzy Analytic Hierarchy Process (FAHP) and the Weighted Aggregated Sum-Product Assessment (WASPAS) methods.

For the problem of optimal selection in multiple manufacturing clouds in (Yang et al., 2020) a quantified approach, based on the AHP and big data, is proposed. Three kinds of uncertainties (of the service market, service execution and the user side respectively) are considered. A novel customer-centric Methodology for Optimal Service Selection in a cloud environment is presented in (Hussain et al., 2020a) and a Fuzzy Linear Best Worst Method for cloud service selection is developed in (Hussain et al., 2020b). The paper (Xu et al., 2020) combined an item-based recommendation program with the Hesitant Fuzzy Linguistic Multi-criteria Analysis of Preferences to analyse hesitant fuzzy linguistic information.

The set of QoS criteria is considered as the most significant factor for the selection of an appropriate CSP. The CSP selection problem is a complex multicriteria decision problem for a new cloud customer. Many of the QoS criteria that must be considered in the problem of CSP selection are both quantitative and qualitative criteria. This fact determines the inclusion in the decision process of the uncertainty due to the subjectivism of the qualitative criteria evaluation.

In the present paper a new multicriteria framework based on the Matter Element Extension (MEE) method is proposed to solve the problem of ranking a set of CSPs and the selection of the CSP that best meets the requirements of an organization. MEE method is defined as a multiple criteria decision method which is ideal to solve multiple criteria problems by formalizing the decision process. It can be enhanced by combining it with weighting methods. The main steps of the MEE method are presented in the following. First, the decision process is defined and the problem is divided into matter elements (objects). Then the evaluation criteria are selected. The set of appreciation degrees and the classic and extended intervals are defined for each criterion. Then the degree of correlation for each criterion is calculated. Finally, the degree of integrated correlation of the matter elements objects is calculated by integration methods. The appreciation degree (which includes the maximum degree of integrated correlation) defines the degree to which the problem element falls within the required conditions. The multicriteria framework and MEE method will be implemented for ranking a 17 CSPs set evaluated according to 9 QoS criteria. A sensitivity analysis will also be performed by varying the endpoints of the extended intervals for the involved criteria. The solutions and the CSP ranks obtained by the MEE method will be analysed.

This paper is organized as follows. In the second section a multicriteria framework based on MEE method for the CSPs selection is proposed. Section three is devoted to the presentation of the multicriteria MEE method. In the fourth section the multicriteria framework is implemented in a case study and the results are analysed. In the fifth section a sensitivity analysis is performed. The paper ends with a section dedicated to conclusions.

\section{A New Multicriteria Framework for CSPs Selection Decision Process}

Purchasing cloud services requires for an organization to choose a CSP from a given set of CSPs. Therefore, the objective is to choose the CSP that best meets the requirements of the organization. From the multitude of CSPs, available on the market, using a recognized database with measured CSPs characteristics quality data, with or without the help of a cloud broker, the organization must choose a CSP that meets its requirements. This problem can be solved with the help of a multicriteria decision method.

To solve the objective of choosing the best CSP a new framework based on the MEE method will be proposed. Within this framework, several stages are established. In the first stage, the

https://www.sic.ici.ro 
input data in the MEE method are defined and constructed: the set of CSPs, the set of criteria, the set of appreciation degrees, the classical domain intervals, the extended domain intervals and the evaluation matrix. In the second stage, a weighting method or a combination of weighting methods is applied to calculate the weights of the criteria. The entropy method is selected for criteria weighting. Then the MEE method is applied and a ranking of CSPs is obtained. A sensitivity analysis is performed by modifying the classical or extended intervals and studying the obtained results. A synthetic presentation of the MEE multicriteria framework is illustrated in Figure 1.

\subsection{Input Data}

A matter-element $R$ is the logical unit of matterelement theory, $R=(N, C, V)$. $N, C$ and $V$ are called the three fundamental elements of matter-element: $P$ is the matter name, $C$ is the set of matter features (the set of criteria) and $V$ is a set of values or intervals values.

The input data in the MEE method are:

1. Decision problem $P$ : choosing the CSP that best meets the requirements,

2. Criteria set: $C=\left\{c_{1}, c_{2}, \ldots, c_{n}\right\}$,

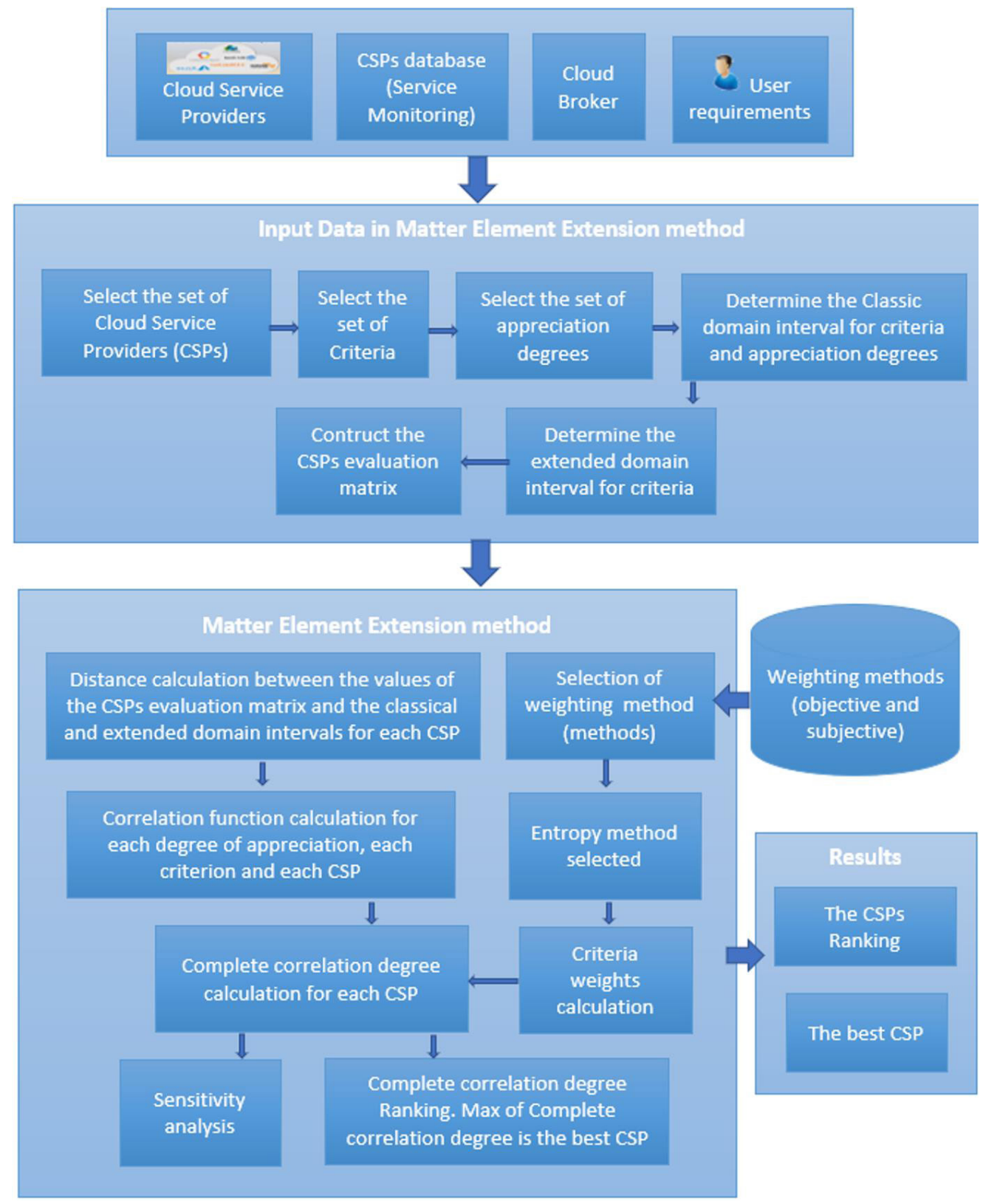

Figure 1. MEE Multicriteria framework architecture 
3. CSPs set: $A=\left\{A_{1}, A_{2}, \ldots, A_{m}\right\}$,

4. Appreciation degrees set: $M=\left\{m_{1}, m_{2}, \ldots, m_{1}\right\}$. The set $M$ is an ordered set. Appreciation grades can be numeric or alphanumeric. For example, $m_{1}=$ "Very Good", $m_{2}=$ "Good", $m_{3}=$ "Medium", $m_{4}=$ "Poor", $m_{5}=$ "Very Poor". Their order is descending. For numerical values their order is $m_{1}>m_{2}>\ldots>m_{1}$,

5. Classic domain intervals for criteria and appreciation degrees,

$v_{j k}^{C}=\left\langle a_{j k}, b_{j k}\right\rangle ; j=1,2, \ldots, n ; k=1,2, \ldots, l$

- the matter element $P_{k}^{C}$ is defined. The start and end values of the classical intervals are defined, for each criterion and each degree of appreciation:

$$
\begin{aligned}
& P_{k}^{C}=\left(f_{k}^{C}, C, V_{k}^{C}\right)=\left[\begin{array}{ccc}
f_{k}^{C} & c_{1} & v_{1 k}^{C} \\
& c_{2} & v_{2 k}^{C} \\
& \ldots & \ldots \\
& c_{n} & v_{n k}^{C}
\end{array}\right]= \\
& =\left[\begin{array}{ccc}
f_{k}^{C} & c_{1} & \left\langle a_{1 k}, b_{1 k}\right\rangle \\
& c_{2} & \left\langle a_{2 k}, b_{2 k}\right\rangle \\
\ldots & \ldots \\
& c_{n} & \left\langle a_{n k}, b_{n k}\right\rangle
\end{array}\right], k=1,2, \ldots, l
\end{aligned}
$$

6. Based on the criteria and value range of $P_{k}^{C}$, the extended domain can be defined. $P^{E}$ represents all the appreciation degrees of the objects to be evaluated. Extended domain intervals for criteria

$v_{j}^{E}=\left\langle d_{j}, e_{j}\right\rangle, j=1,2, \ldots, n$ - the matter element $P^{E}$ are:

$$
\begin{aligned}
& P^{E}=\left(f^{E}, C, V^{E}\right)=\left[\begin{array}{ccc}
f^{E} & c_{1} & v_{1}^{E} \\
& c_{2} & v_{2}^{E} \\
& \ldots & \ldots \\
& c_{n} & v_{n}^{E}
\end{array}\right]= \\
& =\left[\begin{array}{ccc}
f^{E} & c_{1} & \left\langle d_{1}, e_{1}\right\rangle \\
& c_{2} & \left\langle d_{2}, e_{2}\right\rangle \\
\ldots & \ldots \\
& c_{n} & \left\langle d_{n}, e_{n}\right\rangle
\end{array}\right]
\end{aligned}
$$

All intervals $\left\langle a_{j k}, b_{j k}\right\rangle$ are included in the intervals $\left\langle d_{j}, e_{j}\right\rangle, j=1,2, \ldots, n$

The evaluation matrix $X=\left(x_{i j}\right)$

is constructed. It is a $n \times m$ matrix. The matter elements $X_{j}$ corresponding to the columns of the evaluation matrix $X=\left(x_{i j}\right)$ are:

$$
X=\left(f_{j}, C, X_{j}\right)=\left[\begin{array}{ccc}
f_{j} & c_{1} & x_{1 j} \\
& c_{2} & x_{2 j} \\
& \ldots & \ldots \\
& c_{n} & x_{n j}
\end{array}\right]
$$

$$
j=1,2, \ldots, m
$$

\section{Matter Element Extension Method}

The theory of matter-element was first established by Cai (1983 and 1999) and has now become a research method applied in many fields. This method combines qualitative analysis with quantitative research while dealing with complex multi-criteria objective things. MEE method has been applied in many domains. Some recent areas of application of the MEE method are: suitability of land for agriculture (Seyedmohammadi et al., 2019), suitability for regional biomass power generation development (Song et al., 2020), sustainability of water-energy-food (Wang et al., 2018), shale gas industry sustainability (Wang $\& \mathrm{Li}, 2019$ ), evaluation of regional integrated energy systems (Wang et al., 2020), tunnel retro-reflective arch in an extra-long tunnel (Zhao et al., 2021), rock burst, disaster in deep underground engineering (Chen et al., 2019), cloud manufacturing (Zhu et al., 2018), wind farm site selection (Tan et al., 2020), enterprise innovation capability (Zhang \& Yue, 2017).

Once the input data has been defined, the steps of the MEE method are:

Step 1. Criteria weights calculation. The most critical input to the most MCDM methods is the assignment of criteria weights which can be based on subjective, objective, or a combination of weighting methods. Some more often used weighting methods are: Entropy method (Shannon, 1948), Simple Multi-Attribute Rating Technique (SMART) (Edwards, 1977), Analytical Hierarchy Process (AHP) (Saaty, 1977, 1980), Step-Wise Weight Assessment Ratio Analysis (SWARA) (Kersuliene et al., 2010), Weighted Aggregated 
Sum Product Assessment (WASPAS) (Zavadskas et al., 2012), KEmeny Median Indicator Ranks Accordance (KEMIRA) (Krylovas et al., 2014), Best-Worst Method (BWM) (Rezaei, 2015). A synthesis of criteria weighting methods is presented in (Radulescu \& Radulescu, 2018). This paper specifies 29 weighting methods ordered by the year of publication. For each method, the type of method is specified: objective or subjective, the year of publication and the author(s) of the method (by appropriate reference).

The set of weights $W=\left\{w_{1}, w_{2}, \ldots, w_{\mathrm{n}}\right\}$, for criteria $C=\left\{c_{1}, c_{2}, \ldots, c_{n}\right\}$ can be determined by using a weighting method.

For the present framework Entropy method is selected for criteria weights calculation. The criteria set weights is: $W=\left\{w_{1}, w_{2}, \ldots, w_{\mathrm{n}}\right\}$ where $\sum_{j=1}^{n} w_{j}=1$.

Step 2. The distance between the values of the evaluation matrix $X$ and the classical intervals for each criterion and for each degree of appreciation is calculated.

For every: $i=1,2, \ldots, m ; j=1,2, \ldots, n ; k=1,2, \ldots, l$ the distance is:

$$
\rho\left(x_{i j},\left\langle a_{j k}, b_{j k}\right\rangle\right)=\left|x_{i j}-\frac{a_{j k}+b_{j k}}{2}\right|-\frac{\left(b_{j k}-a_{j k}\right)}{2}
$$

Step 3. The distance between the values of the evaluation matrix $X$ and the extended intervals for each criterion is calculated.

For every $i=1,2, \ldots, m ; j=1,2, \ldots, n ; k=1,2, \ldots, l$ the distance is:

$\rho\left(x_{i j},\left\langle d_{j}, e_{j}\right\rangle\right)=\left|x_{i j}-\frac{d_{j}+e_{j}}{2}\right|-\frac{\left(e_{j}-d_{j}\right)}{2}$

Step 4. The correlation function, necessary to determine the degree of correlation between evaluations is obtained.

For every $i=1,2, \ldots, m ; j=1,2, \ldots, n ; k=1,2, \ldots, l$ the correlation function is $r_{i j k}\left(x_{i j}\right)=$

$$
\left\{\begin{array}{l}
\frac{-\rho\left(x_{i j},\left\langle a_{j k}, b_{j k}\right\rangle\right)}{\left|b_{j k}-a_{j k}\right|}, x_{i j} \in\left\langle a_{j k}, b_{j k}\right\rangle \\
\frac{\rho\left(x_{i j},\left\langle a_{j k}, b_{j k}\right\rangle\right)}{\rho\left(x_{i j},\left\langle d_{j}, e_{j}\right\rangle\right)-\rho\left(x_{i j},\left\langle a_{j k}, b_{j k}\right\rangle\right)}, x_{i j} \notin\left\langle a_{j k}, b_{j k}\right\rangle
\end{array}\right.
$$

A tri-dimensional matrix $R=\left(r_{i j k}\right)$ is obtained.
Step 5. The degree of integrated correlation of the evaluation scheme is thus calculated.

The integrated correlation is:

$r_{i k}^{*}=\sum_{j=1}^{n} w_{j} r_{i j k}$

A matrix $R^{*}=\left(r_{i k}^{*}\right)$ is obtained.

Step 6. For each CSP the level of belonging to the degrees of appreciation is calculated according to the following formula:

$y_{i}=\max _{1 \leq k \leq l}\left(r_{i k}^{*}\right), i=1,2, \ldots, m$

Step 7. The arrays of vector $Y=\left(y_{1}, y_{2}, \ldots, y_{m}\right)$ are ranked in decreasing order. A CSPs ranking is obtained according to the value and the degree of the appreciation obtained.

Let $\sigma:\{1,2, \ldots, m\} \rightarrow\{1,2, \ldots, m\}$ be a permutation such that $y_{\sigma(1)} \geq y_{\sigma(2)} \geq \ldots \geq y_{\sigma(m)}$.

The best CSP is $A_{\sigma(1)}$.

\section{Case Study: Ranking a CSPs Set}

The decision problem $\mathrm{P}$ is to establish a ranking in a given CSPs set and the selection of the CSP which can fulfil the requirements.

The MEE method will be implemented for ranking a set of 17 CSPs evaluated according to 9 QoS criteria.

The CSPs data from the studied set will be built on the basis of data selected from a Cloud Armor dataset (a real-time trust feedback dataset) (Cloud Armor, 2015). Cloud Armor developed a cloud service crawler engine that collected cloud services available on the Web. It managed to identify and collect metadata for about 6,000 real cloud services. The values of trust feedback of QoS criteria range from 0 (insignificant) to 5 (significant). The present case study comprises trust feedback values from cloud users for 9 criteria and 17 CSPs.

The set of cloud providers is composed of: AgileIT (CSP1), AmazonEC4 (CSP2), Backup Genie (CSP3), BlueHost (CSP4), Carbonite (CSP5), ComputerSolutionsEast (CSP6), Dropbox (CSP7), Elephant Drive (CSP8), GoGrid (CSP9), Google online backup (CSP10), HostGator (CSP11), idrive (CSP12), iPage (CSP13), JustHost (CSP14), Open Drive (CSP15), WebHostingHub (CSP16), Zip Cloud (CSP17). 
The QoS criteria taken into account are (the set C): Availability (C1), Trust result (C2), Customer Service (C3), Technical_Support (C4), Ease_Of_ Use (C5), Price (C6), Speed (C7), Response Time (C8), Storage_Space (C9). The scale for evaluation is $[0,5]$. The criteria are organized on a single level so that there are no subcriteria.

The appreciation degrees that will be used to define the classical and extended domain intervals and the evaluation of each CSP are defined. The set $M$ of the appreciation degrees contains 5 elements and is defined as follows: $\mathrm{M}=\{$ very good, good, medium, weak, very weak\}.

The trust feedback of QoS criteria, from the built data set, is the evaluation matrix $X=\left(x_{i j}\right)$, $i=1,2, \ldots, 17, j=1,2, \ldots, 9$ (Table 1$)$.

CSPs appreciation degree for a criterion is obtained by checking the membership of the score at an interval from a given set of intervals.

The classical domain intervals for the appreciation degrees are then defined. Thus, for each appreciation degree, from the set $\mathrm{M}$, and for each criterion, from the set $C$, the beginning and end values of the classical intervals are defined. For the starting value of the classical domain interval, for each criterion and the degree "very good", the maximum value from the evaluation matrix, was considered. For the end value of the classical domain interval, for each criterion and degree "very weak", the minimum value from the evaluation matrix, was considered. Then the classical domain intervals were divided equally, by degrees, for each criterion. These classic domain intervals, for each grade and criterion, are presented in Table 2.

The extended domain intervals are defined for the set of criteria $\mathrm{C}$. It consists of the intervals of all possible values for the criteria. The start and end values of the extended intervals for each criterion, in set $\mathrm{C}$, are defined. These values are 0 and 5 , the interval for each criterion in $\mathrm{C}$ is $[0$; 5]. Since the scale of CSPs evaluation is the same for each criterion, the normalization is not taken into consideration.

The criteria weights are calculated by the entropy method (Table 3).

The MEE method is applied. The distances between the elements of the evaluation matrix $\mathrm{X}$ and the classical domain intervals for each CSP, for each criterion and each degree of appreciation, are calculated according to formula (4). The distances between the elements of the evaluation matrix $\mathrm{X}$ and the extended domain intervals

Table 1. Evaluation matrix $X$

\begin{tabular}{|c|c|c|c|c|c|c|c|c|c|}
\hline \multirow{2}{*}{ CSPs } & \multicolumn{9}{|c|}{ Criteria } \\
\hline & $\mathrm{C} 1$ & $\mathrm{C} 2$ & $\mathrm{C} 3$ & $\mathrm{C} 3$ & C5 & C6 & $\mathrm{C} 7$ & C8 & C9 \\
\hline CSP1 & 3.77 & 4.51 & 4.51 & 4.56 & 3.10 & 4.41 & 3.98 & 4.57 & 2.88 \\
\hline CSP2 & 4.00 & 4.00 & 3.19 & 3.00 & 3.10 & 3.00 & 3.98 & 4.08 & 2.88 \\
\hline CSP3 & 3.77 & 4.00 & 4.00 & 4.00 & 4.00 & 4.00 & 3.98 & 4.08 & 4.00 \\
\hline CSP4 & 2.76 & 3.03 & 3.19 & 2.98 & 2.75 & 3.56 & 3.98 & 4.08 & 2.88 \\
\hline CSP5 & 2.38 & 2.69 & 2.44 & 2.47 & 3.14 & 2.65 & 2.58 & 2.70 & 3.47 \\
\hline CSP6 & 3.77 & 4.83 & 4.83 & 5.00 & 3.10 & 4.33 & 3.98 & 5.00 & 2.88 \\
\hline CSP7 & 2.91 & 2.93 & 2.93 & 2.68 & 2.95 & 2.90 & 3.00 & 3.00 & 2.88 \\
\hline CSP8 & 3.77 & 2.70 & 2.70 & 2.60 & 2.60 & 2.70 & 3.98 & 4.08 & 2.80 \\
\hline CSP9 & 4.00 & 4.00 & 3.19 & 4.40 & 3.10 & 4.20 & 3.98 & 4.08 & 2.88 \\
\hline CSP10 & 3.77 & 2.25 & 1.75 & 1.50 & 2.00 & 0.75 & 3.98 & 4.08 & 1.50 \\
\hline CSP11 & 4.39 & 4.38 & 4.75 & 4.23 & 3.52 & 4.42 & 4.72 & 4.08 & 2.88 \\
\hline CSP12 & 4.33 & 2.64 & 2.36 & 2.45 & 2.91 & 1.73 & 4.33 & 4.67 & 2.27 \\
\hline CSP13 & 3.80 & 3.91 & 3.19 & 4.00 & 3.74 & 3.88 & 3.98 & 4.08 & 2.88 \\
\hline CSP14 & 3.59 & 3.39 & 1.00 & 3.35 & 3.52 & 4.02 & 3.98 & 4.00 & 2.88 \\
\hline CSP15 & 3.77 & 1.25 & 1.00 & 1.25 & 1.50 & 1.50 & 3.98 & 4.08 & 1.50 \\
\hline CSP16 & 4.73 & 4.63 & 4.73 & 4.50 & 3.10 & 4.51 & 4.71 & 4.08 & 2.88 \\
\hline CSP17 & 4.63 & 4.61 & 4.46 & 4.46 & 4.52 & 4.62 & 4.56 & 4.59 & 4.65 \\
\hline Min & 2.38 & 1.25 & 1.00 & 1.25 & 1.50 & 0.75 & 2.58 & 2.70 & 1.50 \\
\hline Max & 4.73 & 4.83 & 4.83 & 5.00 & 4.52 & 4.62 & 4.72 & 5.00 & 4.65 \\
\hline
\end{tabular}

https://www.sic.ici.ro 
Table 2. Classic domain intervals

\begin{tabular}{cccccc}
\hline \multirow{2}{*}{ Criteria } & \multicolumn{5}{c}{ Appreciation degree } \\
\cline { 2 - 6 } & very good & good & medium & weak & very weak \\
\hline C1 & {$[4.27-4.73]$} & {$[3.80-4.26]$} & {$[3.33-3.79]$} & {$[2.86-3.32]$} & {$[2.38-2.85]$} \\
C2 & {$[4.13-4.83]$} & {$[3.41-4.12]$} & {$[2.69-3.40]$} & {$[1.98-2.68]$} & {$[1.25-1.97]$} \\
C3 & {$[4.06-4.83]$} & {$[3.29-4.07]$} & {$[2.52-3.30]$} & {$[1.76-2.53]$} & {$[1.00-1.77]$} \\
C4 & {$[4.24-5.00]$} & {$[3.49-4.25]$} & {$[2.74-3.50]$} & {$[1.99-2.75]$} & {$[1.25-2.00]$} \\
C5 & {$[3.91-4.52]$} & {$[3.30-3.92]$} & {$[2.70-3.31]$} & {$[2.09-2.71]$} & {$[1.50-2.10]$} \\
C6 & {$[3.84-4.62]$} & {$[3.06-3.85]$} & {$[2.29-3.07]$} & {$[1.51-2.30]$} & {$[0.75-1.52]$} \\
C7 & {$[4.29-4.72]$} & {$[3.86-4.30]$} & {$[3.43-3.87]$} & {$[3.00-3.44]$} & {$[2.58-3.01]$} \\
C8 & {$[4.53-5.00]$} & {$[4.07-4.54]$} & {$[3.61-4.08]$} & {$[3.15-3.62]$} & {$[2.70-3.16]$} \\
C9 & {$[4.01-4.65]$} & {$[3.38-4.02]$} & {$[2.75-3.39]$} & {$[2.12-2.76]$} & {$[1.50-2.13]$} \\
\hline
\end{tabular}

Table 3. The weights of criteria

\begin{tabular}{cccccccccc}
\hline Criteria & & \multicolumn{1}{c}{ Criteria } \\
\cline { 3 - 10 } Weights & C1 & C2 & C3 & C3 & C5 & C6 & C7 & C8 & C9 \\
\hline $\mathrm{W}$ & 0.116 & 0.110 & 0.103 & 0.108 & 0.113 & 0.105 & 0.116 & 0.116 & 0.112 \\
\hline
\end{tabular}

for each CSP, for each criterion, are calculated, according to formula (5).

The correlation function is calculated for each degree of appreciation, for each criterion and for each CSP according to equation (6). The correlation function calculated for the first CSP is presented in Table 4.

The integrated correlation degree is calculated according to formula (7) and the values obtained are presented in Table 5.

Table 4. The correlation function values for CSP1

\begin{tabular}{|c|c|c|c|c|c|}
\hline \multirow{2}{*}{ Criteria } & \multicolumn{5}{|c|}{ Appreciation degree } \\
\hline & very good & good & medium & weak & very weak \\
\hline $\mathrm{C} 1$ & -0.289 & -0.0238 & 0.0435 & -0.2679 & -0.4279 \\
\hline $\mathrm{C} 2$ & 0.4571 & -0.4432 & -0.6937 & -0.7888 & -0.8383 \\
\hline $\mathrm{C} 3$ & 0.4156 & -0.4731 & -0.7118 & -0.8016 & -0.8483 \\
\hline $\mathrm{C} 4$ & 0.4211 & -0.4133 & -0.7067 & -0.8044 & -0.8533 \\
\hline $\mathrm{C} 5$ & -0.2989 & -0.0952 & 0.3443 & -0.1703 & -0.3448 \\
\hline $\mathrm{C} 6$ & 0.2692 & -0.487 & -0.6943 & -0.7815 & -0.8305 \\
\hline $\mathrm{C} 7$ & -0.2331 & 0.2727 & -0.0973 & -0.3462 & -0.4874 \\
\hline $\mathrm{C} 8$ & 0.0851 & -0.0652 & -0.5326 & -0.6884 & -0.7663 \\
\hline $\mathrm{C} 9$ & -0.3477 & -0.1908 & 0.2031 & -0.0536 & -0.2613 \\
\hline \multicolumn{6}{|c|}{ Table 5. The integrated correlation degree } \\
\hline \multirow{2}{*}{ CSPs } & \multicolumn{5}{|c|}{ Appreciation degree } \\
\hline & very good & good & medium & weak & very weak \\
\hline CSP1 & 0.04351 & -0.20423 & -0.30562 & -0.51520 & -0.62294 \\
\hline CSP2 & -0.28127 & 0.04125 & 0.04990 & -0.28068 & -0.44493 \\
\hline CSP3 & -0.10084 & 0.07609 & -0.26549 & -0.48612 & -0.60350 \\
\hline CSP4 & -0.32084 & -0.05228 & 0.07181 & -0.19638 & -0.32832 \\
\hline CSP5 & -0.37712 & -0.22004 & -0.03542 & -0.07487 & -0.19279 \\
\hline CSP6 & -0.09417 & -0.44437 & -0.42866 & -0.59794 & -0.68535 \\
\hline CSP7 & -0.36943 & -0.21929 & 0.10858 & -0.05555 & -0.16070 \\
\hline CSP8 & -0.33770 & -0.10793 & 0.06736 & -0.09435 & -0.32356 \\
\hline CSP9 & -0.13767 & 0.01190 & -0.12256 & -0.39442 & -0.53145 \\
\hline CSP10 & -0.48743 & -0.30219 & -0.25572 & -0.18527 & -0.11911 \\
\hline CSP11 & 0.00504 & -0.22630 & -0.41779 & -0.58099 & -0.67638 \\
\hline CSP12 & -0.21392 & -0.23756 & -0.20008 & -0.10713 & -0.36349 \\
\hline CSP13 & -0.21773 & 0.10554 & -0.11897 & -0.37647 & -0.51842 \\
\hline CSP14 & -0.29353 & -0.06926 & -0.02403 & -0.31111 & -0.41009 \\
\hline CSP15 & -0.53344 & -0.35709 & -0.32371 & -0.30069 & -0.16160 \\
\hline CSP16 & -0.01180 & -0.40472 & -0.43639 & -0.61029 & -0.69850 \\
\hline CSP17 & 0.19867 & -0.45394 & -0.69183 & -0.78416 & -0.83372 \\
\hline
\end{tabular}


Table 6. MEE solution and CSPs ranks extension

\begin{tabular}{lccccc}
\hline CSPs & Solutions & Ranks & CSPs & Solutions & Ranks \\
\hline CSP1 & 0.0435 & 8 & CSP10 & -0.1191 & 16 \\
CSP2 & 0.0499 & 7 & CSP11 & 0.0050 & 10 \\
CSP3 & 0.0761 & 4 & CSP12 & -0.1071 & 15 \\
CSP4 & 0.0718 & 5 & CSP13 & 0.1055 & 3 \\
CSP5 & -0.0354 & 13 & CSP14 & -0.0240 & 12 \\
CSP6 & -0.0942 & 14 & CSP15 & -0.1616 & 17 \\
CSP7 & 0.1086 & 2 & CSP16 & -0.0118 & 11 \\
CSP8 & 0.0674 & 6 & CSP17 & 0.1987 & 1 \\
CSP9 & 0.0119 & 9 & & & \\
\hline
\end{tabular}

The maximum for each CSP is calculated according to formula (8), (Table 6). Then the ranks are obtained.

By ranking these values, the CSPs ranks are obtained. Thus, the best CSP is CSP17 followed by CSP7 and the last is CSP15.

The case study was solved with a set of MATLAB scripts that implement the MEE method.

\section{Sensitivity Analysis}

The endpoints of the extended domain ranges will be varied and the MEE solutions and the ranks obtained for 16 cases will be analysed. The same input data in the MEE method as in the case study presented above (criteria set, CSPs set, set of degrees of appreciation, classical domain intervals, evaluations matrix, weights associated with criteria) will be kept.

Consider the four values $\mathrm{d}, \mathrm{a}, \mathrm{b}, \mathrm{e}$ in Figure 2.

Figure 2 corresponds to a single criterion in the set $\mathrm{C}$.
For the 9 criteria a vector $\mathbf{a}=(\mathrm{a} 1, \mathrm{a} 2, \ldots ., \mathrm{a} 9)$ and a vector $\mathbf{b}=(\mathrm{b} 1, \mathrm{~b} 2, \ldots ., \mathrm{b} 9)$ will be considered for the classical domain intervals (Table 7) and 2 vectors of 9 equal components $\mathbf{d}=(\mathrm{d}, \mathrm{d}, \ldots, \mathrm{d})$ and $\mathbf{e}=(\mathrm{e}, \mathrm{e}, \ldots, \mathrm{e})$ for the extended domain intervals will be considered. The intervals end points [d; e] of the $[0 ; 5]$ will be varied by decreasing the distance of $\mathbf{d}$ from a (with step 0.15 ) and of $\mathbf{e}$ from $\mathbf{b}$ (with step 0.15). An interval matrix (matrix whose arrays are extended domain intervals) is obtained (Table 8).

For each interval matrix array, the MEE method is applied and the CSPs ranking is obtained (Table 9).

There is a variation of the obtained ranks. The Spearman Correlation of the CSPs ranks is calculated (Table 10).

Note that the differences between the ranks is small. All the arrays of the Spearman correlation matrix are in the interval $[0.98 ; 1]$. This shows that the variation of the end points of the extended domain interval $[\mathbf{d} ; \mathbf{e}]$ has a small impact on the CSPs rankings.

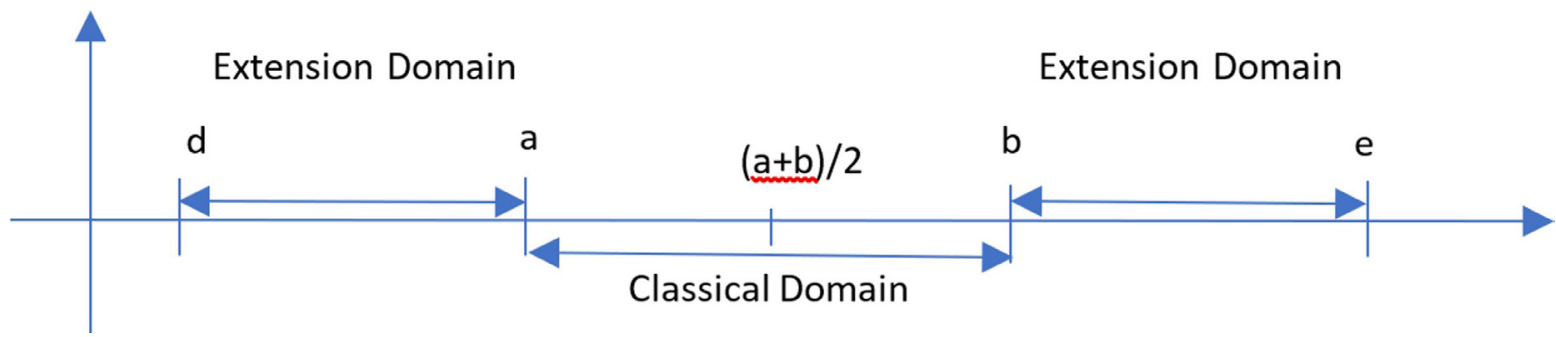

Figure 2. The classical and extension domain 
Table 7. Classical domain intervals for criteria

\begin{tabular}{cccccccccccc}
\hline Classical & & \multicolumn{8}{c}{ Criteria } & \multirow{2}{*}{ min } & \multirow{2}{*}{ max } \\
\cline { 3 - 10 } Domain & C1 & C2 & C3 & C3 & C5 & C6 & C7 & C8 & C9 & & \\
\hline $\mathbf{a}$ & 2.38 & 1.25 & 1.00 & 1.25 & 1.50 & 0.75 & 2.58 & 2.70 & 1.50 & 0.75 & 2.70 \\
$\mathbf{b}$ & 4.73 & 4.83 & 4.83 & 5.00 & 4.52 & 4.62 & 4.72 & 5.00 & 4.65 & 4.52 & 5.00 \\
\hline
\end{tabular}

Table 8. Extended domain intervals

\begin{tabular}{cccc}
\hline$[0 ; 5]$ & {$[0 ; 4.85]$} & {$[0 ; 4.7]$} & {$[0 ; 4.55]$} \\
{$[0.15 ; 5]$} & {$[0.15 ; 4.85]$} & {$[0.15 ; 4.7]$} & {$[0.15 ; 4.55]$} \\
{$[0.3 ; 5]$} & {$[0.3 ; 4.85]$} & {$[0.5 ; 4.7]$} & {$[0.3 ; 4.55]$} \\
{$[0.45 ; 5]$} & {$[0.45 ; 4.85]$} & {$[0.45 ; 4.7]$} & {$[0.45 ; 4.55]$} \\
\hline
\end{tabular}

Table 9. The MEE ranks

\begin{tabular}{ccccccccccccccccc}
\hline CSPs & $\mathbf{1}$ & $\mathbf{2}$ & $\mathbf{3}$ & $\mathbf{4}$ & $\mathbf{5}$ & $\mathbf{6}$ & $\mathbf{7}$ & $\mathbf{8}$ & $\mathbf{9}$ & $\mathbf{1 0}$ & $\mathbf{1 1}$ & $\mathbf{1 2}$ & $\mathbf{1 3}$ & $\mathbf{1 4}$ & $\mathbf{1 5}$ & $\mathbf{1 6}$ \\
\hline CSP1 & 8 & 8 & 8 & 8 & 8 & 8 & 8 & 8 & 8 & 8 & 8 & 8 & 8 & 8 & 8 & 8 \\
CSP2 & 7 & 7 & 7 & 7 & 7 & 7 & 7 & 7 & 7 & 7 & 7 & 7 & 7 & 7 & 7 & 7 \\
CSP3 & 4 & 4 & 4 & 4 & 4 & 4 & 4 & 4 & 4 & 4 & 4 & 4 & 4 & 4 & 4 & 4 \\
CSP4 & 5 & 5 & 6 & 6 & 5 & 5 & 6 & 6 & 5 & 5 & 6 & 6 & 5 & 5 & 6 & 6 \\
CSP5 & 13 & 13 & 13 & 12 & 13 & 13 & 13 & 12 & 13 & 13 & 12 & 12 & 13 & 13 & 12 & 12 \\
$\operatorname{CSP} 6$ & 14 & 14 & 14 & 14 & 14 & 14 & 14 & 14 & 14 & 14 & 14 & 14 & 14 & 14 & 14 & 14 \\
$\operatorname{CSP} 7$ & 2 & 2 & 3 & 3 & 2 & 2 & 3 & 3 & 2 & 2 & 3 & 3 & 2 & 2 & 3 & 3 \\
$\operatorname{CSP} 8$ & 6 & 6 & 5 & 5 & 6 & 6 & 5 & 5 & 6 & 6 & 5 & 5 & 6 & 6 & 5 & 5 \\
$\operatorname{CSP} 9$ & 9 & 9 & 10 & 11 & 9 & 9 & 10 & 11 & 9 & 9 & 10 & 11 & 9 & 9 & 10 & 11 \\
$\operatorname{CSP} 10$ & 16 & 16 & 15 & 15 & 16 & 16 & 15 & 15 & 16 & 16 & 15 & 15 & 16 & 16 & 15 & 15 \\
$\operatorname{CSP} 11$ & 10 & 10 & 9 & 9 & 10 & 10 & 9 & 9 & 10 & 10 & 9 & 9 & 10 & 10 & 9 & 9 \\
$\operatorname{CSP} 12$ & 15 & 15 & 16 & 16 & 15 & 15 & 16 & 16 & 15 & 15 & 16 & 16 & 15 & 15 & 16 & 16 \\
$\operatorname{CSP} 13$ & 3 & 3 & 2 & 2 & 3 & 3 & 2 & 2 & 3 & 3 & 2 & 2 & 3 & 3 & 2 & 2 \\
$\operatorname{CSP} 14$ & 12 & 12 & 12 & 13 & 12 & 12 & 12 & 13 & 12 & 12 & 13 & 13 & 12 & 12 & 13 & 13 \\
$\operatorname{CSP} 15$ & 17 & 17 & 17 & 17 & 17 & 17 & 17 & 17 & 17 & 17 & 17 & 17 & 17 & 17 & 17 & 17 \\
$\operatorname{CSP} 16$ & 11 & 11 & 11 & 10 & 11 & 11 & 11 & 10 & 11 & 11 & 11 & 10 & 11 & 11 & 11 & 10 \\
$\operatorname{CSP} 17$ & 1 & 1 & 1 & 1 & 1 & 1 & 1 & 1 & 1 & 1 & 1 & 1 & 1 & 1 & 1 & 1 \\
\hline
\end{tabular}

Table 10. The Spearman correlation matrix

\begin{tabular}{|c|c|c|c|c|c|c|c|c|c|c|c|c|c|c|c|}
\hline 1 & 1 & 0.99 & 0.98 & 1 & 1 & 0.99 & 0.98 & 1 & 1 & 0.99 & 0.98 & 1 & 1 & 0.99 & 0.98 \\
\hline 1 & 1 & 0.99 & 0.98 & 1 & 1 & 0.99 & 0.98 & 1 & 1 & 0.99 & 0.98 & 1 & 1 & 0.99 & 0.98 \\
\hline 0.99 & 0.99 & 1 & 1 & 0.99 & 0.99 & 1 & 1 & 0.99 & 0.99 & 1 & 1 & 0.99 & 0.99 & 1 & 1 \\
\hline 0.98 & 0.98 & 1 & 1 & 0.98 & 0.98 & 1 & 1 & 0.98 & 0.98 & 1 & 1 & 0.98 & 0.98 & 1 & 1 \\
\hline 1 & 1 & 0.99 & 0.98 & 1 & 1 & 0.99 & 0.98 & 1 & 1 & 0.99 & 0.98 & 1 & 1 & 0.99 & 0.98 \\
\hline 1 & 1 & 0.99 & 0.98 & 1 & 1 & 0.99 & 0.98 & 1 & 1 & 0.99 & 0.98 & 1 & 1 & 0.99 & 0.98 \\
\hline 0.99 & 0.99 & 1 & 1 & 0.99 & 0.99 & 1 & 1 & 0.99 & 0.99 & 1 & 1 & 0.99 & 0.99 & 1 & 1 \\
\hline 0.98 & 0.98 & 1 & 1 & 0.98 & 0.98 & 1 & 1 & 0.98 & 0.98 & 1 & 1 & 0.98 & 0.98 & 1 & 1 \\
\hline 1 & 1 & 0.99 & 0.98 & 1 & 1 & 0.99 & 0.98 & 1 & 1 & 0.99 & 0.98 & 1 & 1 & 0.99 & 0.98 \\
\hline 1 & 1 & 0.99 & 0.98 & 1 & 1 & 0.99 & 0.98 & 1 & 1 & 0.99 & 0.98 & 1 & 1 & 0.99 & 0.98 \\
\hline 0.99 & 0.99 & 1 & 1 & 0.99 & 0.99 & 1 & 1 & 0.99 & 0.99 & 1 & 1 & 0.99 & 0.99 & 1 & 1 \\
\hline 0.98 & 0.98 & 1 & 1 & 0.98 & 0.98 & 1 & 1 & 0.98 & 0.98 & 1 & 1 & 0.98 & 0.98 & 1 & 1 \\
\hline 1 & 1 & 0.99 & 0.98 & 1 & 1 & 0.99 & 0.98 & 1 & 1 & 0.99 & 0.98 & 1 & 1 & 0.99 & 0.98 \\
\hline 1 & 1 & 0.99 & 0.98 & 1 & 1 & 0.99 & 0.98 & 1 & 1 & 0.99 & 0.98 & 1 & 1 & 0.99 & 0.98 \\
\hline 0.99 & 0.99 & 1 & 1 & 0.99 & 0.99 & 1 & 1 & 0.99 & 0.99 & 1 & 1 & 0.99 & 0.99 & 1 & 1 \\
\hline 0.98 & 0.98 & 1 & 1 & 0.98 & 0.98 & 1 & 1 & 0.98 & 0.98 & 1 & 1 & 0.98 & 0.98 & 1 & 1 \\
\hline
\end{tabular}




\section{Conclusion}

The paper proposes a new multicriteria framework based on the MEE method to solve problem of ranking of a set of CSPs and selection of the best CSP.

The main contributions of this research are described as follows: the multicriteria decision process is defined and formalized; a new framework based on MEE method is proposed; the multicriteria framework is applied in a case study for CSPs ranking and selection; a sensitivity analysis is presented by varying the endpoints of the extended domain intervals for the involved criteria. The solutions and the CSPs ranks obtained by the MEE method are analysed and the differences between the arrays of the Spearman correlation matrix are small. More precisely all the arrays of the Spearman correlation matrix are located in the interval $[0.98 ; 1]$. The set of CSPs in the present study and the data are selected from

\section{REFERENCES}

Abdel-Basset, M., Mohamed, M. \& Chang, V. (2018). NMCDA: A framework for evaluating cloud computing services, Future Generation Computer Systems, 86, 12-29.

Al-Faifi, A., Song, B., Hassan, M. M., Alamri, A. \& Gumaei, A. (2019). A hybrid multi criteria decision method for cloud service selection from Smart data, Future Generation Computer Systems, 93, 43-57.

Alam, K. A., Ahmed, R., Butt, F. S., Kim, S. G. \& Ko, K. M. (2018). An uncertainty-aware integrated fuzzy AHP-WASPAS model to evaluate public cloud computing services, Procedia Computer Science, 130, 504-509.

Cai, W. (1983). The extension set and incompatibility problem, Journal of Scientific Exploration, 1, 610-614.

Cai, W. (1999). Extension theory and its application, Chinese Science Bulletin, 44(17), 1538-1548.

Chen, J., Chen, Y., Yang, S., Zhong, X. \& Han, X. (2019). A prediction model on rock burst intensity grade based on variable weight and matter-element extension, PloS one, 14(6), e0218525.

Cloud Armor (2015). The Project Website. Available at: $<$ https://cs.adelaide.edu.au/ cloudarmor/ds.html $>$, last accessed: 2021, February 5.

Eisa, M., Younas, M., Basu, K. \& Awan, I. (2020). Modelling and Simulation of QoS-Aware Service a database called "Cloud Armor". This database contains real-time trust feedback data for a set of about 6000 CSPs.

Matlab scripts were created to solve the method. As far as we know, the method used in this paper has not been used in the context of selecting a CSP. Starting from this approach and its associated method, a software can be developed, with a friendly interface, to support the user / organization to decide on the criteria and CSPs they consider appropriate, the weights associated with the criteria, the classic and extended intervals.

\section{Acknowledgements}

The research reported in this paper was supported by project PN 19370401 "New solutions for complex problems in current ICT research fields based on modelling and optimization", funded by the Romanian Core Program of the Ministry of Research and Innovation (MCI), 2019-2022.

Selection in Cloud Computing, Simulation Modelling Practice and Theory, 103, 102108.

Edwards, W. (1977). How to use multi attribute utility measurement for social decision-making, IEEE Transactions on Systems, Man, and Cybernetics, 7(5), 326-340.

Filip, F. G., Zamfirescu, C. B. \& Ciurea, C. (2017). Computer-supported collaborative decisionmaking. Cham: Springer International Publishing.

Halabi, T. \& Bellaiche, M. (2018). A broker-based framework for standardization and management of Cloud Security-SLAs, Computers \& Security, 75, 59-71.

Hussain, A., Chun, J. \& Khan, M. (2020a). A novel customer-centric Methodology for Optimal Service Selection (MOSS) in a cloud environment, Future Generation Computer Systems, 105, 562-580.

Hussain, A., Chun, J. \& Khan, M. (2020b). A novel framework towards viable Cloud Service Selection as a Service (CSSaaS) under a fuzzy environment, Future Generation Computer Systems, 104, 74-91.

Kersuliene, V. Zavadskas, E. K. \& Turskis, Z. (2010). Selection of rational dispute resolution method by applying new step wise weight assessment ratio analysis (SWARA), Journal of Business Economics and Management, 11(2), 243-258. 
Krylovas, A., Zavadskas, E., Kosareva, N. \& Dadelo, S. (2014). New KEMIRA method for determining criteria priority and weights in solving MCDM problem, International Journal of Information Technology and Decision Making, 13, 1119-1133.

Mahmoud, M. S. \& Hamdan, M. M. (2018). Fundamental issues in networked control systems, IEEE/CAA Journal of Automatica Sinica, 5(5), 902-922.

Martens, B. \& Teuteberg, F. (2012). Decision-making in cloud computing environments: A cost and riskbased approach, Information Systems Frontiers, 14(4), 871-893.

Radulescu, C. Z. \& Radulescu, M. (2018). Group decision support approach for cloud quality of service criteria weighting, Studies in Informatics and Control, 27(3), 275-284 DOI: 10.24846/v27i3y201803

Rezaei, J. (2015). Best-worst multi-criteria decisionmaking method, Omega, 53, 49-57.

Saaty, T. L. (1977). A scaling method for priorities in hierarchical structures, Journal of mathematical psychology, 15(3), 234-281.

Saaty, T. L. (1980). The Analytic Hierarchy Process. McGraw-Hill Press, NewYork.

Seyedmohammadi, J., Sarmadian, F., Jafarzadeh, A. A. \& McDowell, R. W. (2019). Development of a model using matter element, AHP and GIS techniques to assess the suitability of land for agriculture, Geoderma, 352, 80-95.

Shannon, C. E. (1948). A note on the concept of entropy, Bell System Technical Journal, 27, 379-423.

Song, J., Li, K., Ren, J., Yang, W. \& Liu, X. (2020). Holistic suitability for regional biomass power generation development in China: An application of matter-element extension model, Journal of Environmental Management, 276, 111294.

Tan, Q., Wei, T., Peng, W., Yu, Z. \& Wu, C. (2020). Comprehensive evaluation model of wind farm site selection based on ideal matter element and grey clustering, Journal of Cleaner Production, 272, 122658.

Tiwari, R. K. \& Kumar, R. (2020). A framework for prioritizing cloud services in neutrosophic environment, Journal of King Saud UniversityComputer and Information Sciences. Available online, 27 May 2020.
Wang, Q. \& Li, S. (2019). Shale gas industry sustainability assessment based on WSR methodology and fuzzy matter-element extension model: the case study of China, Journal of Cleaner Production, 226 , 336-348.

Wang, Q., Li, S., He, G., Li, R. \& Wang, X. (2018). Evaluating sustainability of water-energy-food (WEF) nexus using an improved matter-element extension model: A case study of China, Journal of Cleaner Production, 202, 1097-1106.

Wang, Y., Li, F., Yang, J., Zhou, M., Song, F., Zhang, D., Xue, L. \& Zhu, J. (2020). Demand response evaluation of RIES based on improved matter-element extension model, Energy, 212, 118121.

Xia, Y. (2015). Cloud control systems, IEEE/CAA Journal of Automatica Sinica, 2(2), 134-142.

Xu, Z., Chen, H. \& He, Y. (2020). A Recommender System Based on Hesitant Fuzzy Linguistic Information with MAPPACC Approach, Studies in Informatics and Control, 29(2), 145-158. DOI: 10.24846/v29i2y202001

Yang, C., Peng, T., Lan, S., Shen, W. \& Wang, L. (2020). Towards IoT-enabled dynamic service optimal selection in multiple manufacturing clouds, Journal of Manufacturing Systems, 56, 213-226.

Zavadskas, E. K., Turskis, Z., Antucheviciene, J. \& Zakarevicius, A. (2012). Optimization of weighted aggregated sum product assessment, Elektronika ir elektrotechnika, 122(6), 3-6.

Zhang, X. \& Yue, J. (2017). Measurement model and its application of enterprise innovation capability based on matter element extension theory, Procedia Engineering, 174, 275-280.

Zhao, X., Zhang, C., Ju, Y., Li, J., Bian, Y. \& Ma, J. (2021). Evaluation of tunnel retro-reflective arch in an extra-long tunnel based on the matterelement extension method, Accident Analysis \& Prevention, 150, 105913.

Zhu, L. N., Zhao, Y. W., Zhao, C. \& Shen, G. J. (2018). A multidimensional extension-based method for resource performance matching in cloud manufacturing, Concurrent Engineering, 26(3), 276-286. 